\title{
Lineability of Linearly Sensitive Functions
}

\author{
Artur Bartoszewicz, Małgorzata Filipczak, and \\ Małgorzata Terepeta
}

\begin{abstract}
In the paper we will focus on lineability of some subsets of $\mathbb{R}^{[0,1]}$ which are called linearly sensitive. A function $f$ is called linearly sensitive with respect to the property (or condition) $(P)$ if $f$ has the property $(P)$ and for any $a \neq 0$ the function $f+a \cdot$ id does not have the property $(P)$. We discuss some general method of proving $\mathfrak{c}$-lineability and use this method to examine lineability of the family of all continuous functions linearly sensitive to the Luzin $(N)$-property, the family of functions linearly sensitive to the Świątkowski condition and the family of functions linearly sensitive to the strong Świątkowski condition.
\end{abstract}

Mathematics Subject Classification. 46B87, 15A03, 26A15.

Keywords. Lineability, Luzin (N)-property, Świątkowski property, strong Świątkowski property.

\section{Introduction}

Let $\kappa$ be a cardinal number. We say that a subset $M$ of a linear space $V$ is $\kappa$-lineable if $M \cup\{0\}$ contains a linear subspace of dimension $\kappa$. If, additionally, $V$ is an algebra, then in a similar way one can define an algebrability of subset of $V$. For a vector topological space $V$, a subset $M \subset V$ is called spaceable (dense lineable) if $M \cup\{0\}$ contains a closed infinitely dimentional subspace (dense subspace) of $V$. The lineability (algebrability) problem of subsets of function spaces has been studied during previous years by many authors (see $[1,2,16]$ ) and also recently (see $[3,6])$. In the present paper we will focus on $\mathfrak{c}$-lineability of some subsets of $\mathbb{R}^{[0,1]}$. 
The common way of proving $\kappa$-lineability (in particular $\mathfrak{c}$-lineability) of $M$ is to construct a set of cardinality $\kappa$ of linearly independent elements of $M$ and to show that any linear combination of them belongs to $M$.

There are some subsets of $\mathbb{R}^{[0,1]}$ which are extremely nonlinear in certain senses. For example, it is well known that a sum of two Darboux functions can be any arbitrary function in $\mathbb{R}^{[0,1]}$. The same can be said about the class of perfectly everywhere surjective functions. On the other hand, the maximal additive class for Darboux functions consists of constant functions only, which means that for any nonconstant function $f$ there exists a Darboux function $g$ such that $f+g$ is not Darboux.

The classes of linearly sensitive functions with respect to the property $(P)$ are also extremely nonlinear in a very strong sense.

Definition 1. Let $(P)$ be a property of functions in $\mathbb{R}^{[0,1]}$. We say that $f$ is linearly sensitive with respect to $(P)$ if $f$ has the property $(P)$ and for any real $a \neq 0$ the function $f+a \cdot$ id does not have the property $(P)$.

\section{The Main Method}

In the present paper we consider

- continuous functions linearly sensitive with respect to the Luzin $(N)$ property,

- functions linearly sensitive with respect to the Świątkowski condition,

- functions linearly sensitive with respect to the strong Świątkowski condition.

We will prove that all these classes are $\mathfrak{c}$-lineable. The fundamental method to prove it is contained in the following theorem:

Theorem 2. Let $M \subset \mathbb{R}^{[0,1]}$. Assume that there exists a sequence $\left(\left[a_{n}, b_{n}\right]\right)_{n \in \mathbb{N}}$ of closed disjoint intervals contained in $[0,1]$ and a sequence $\left(F_{n}\right)_{n \in \mathbb{N}}$ of functions $F_{n}:\left[a_{n}, b_{n}\right] \rightarrow \mathbb{R}$ such that for any $A \subset \mathbb{N}$ and any bounded nonzero sequence of reals $\left(\alpha_{i}\right)_{i \in A}$, the function

$$
G_{\alpha_{i}, A}(x)= \begin{cases}\alpha_{i} F_{i}(x) & \text { for } x \in\left[a_{i}, b_{i}\right], i \in A \\ 0 & \text { for } x \notin \bigcup_{i \in A}\left[a_{i}, b_{i}\right] .\end{cases}
$$

belongs to $M$. Then $M$ is c-lineable.

An easy proof of Theorem 2 is based on the well known theorem on the cardinality of independent families of sets in $\mathcal{P}(X)$ for an arbitrary set $X$. 
Theorem 3 (Fichtenholz-Kantorovich [8]). For any set $X$ there exists a family $\mathcal{B} \subset \mathcal{P}(X)$ of cardinality $2^{|X|}$ such that for any finite sequences $B_{1}, \ldots, B_{n} \in \mathcal{B}$ and $\varepsilon_{1}, \ldots, \varepsilon_{n} \in\{0,1\}$

$$
B_{1}^{\varepsilon_{1}} \cap \cdots \cap B_{n}^{\varepsilon_{n}} \neq \varnothing
$$

(where $B^{1}=B$ and $\left.B^{0}=X \backslash B\right)$. Moreover all the sets in $\mathcal{B}$ can be chosen with the cardinality $|X|$.

Such a family $\mathcal{B}$ is called an independent one.

Proof of Theorem 2. Let $\mathcal{B}=\left\{B_{\xi}: \xi<\mathfrak{c}\right\}$ be an independent family in $\mathcal{P}(\omega)$ (where $\omega=\{0,1,2, \ldots\}$ ). For any $\xi<\mathfrak{c}$ we put

$$
f_{\xi}(x)= \begin{cases}F_{i}(x) & \text { for } x \in\left[a_{i}, b_{i}\right], i \in B_{\xi} \\ 0 & \text { otherwise. }\end{cases}
$$

By (1), any function $f_{\xi}$ for $\xi<\mathfrak{c}$ belongs to $M$. Moreover, $\left\{f_{\xi}: \xi<\mathfrak{c}\right\}$ is a family of linearly independent functions in $M$. Indeed, suppose that $f_{\xi}=$ $c_{1} f_{\xi_{1}}+\cdots+c_{k} f_{\xi_{k}}$ for some $k \in \mathbb{N}, \xi_{i}<\mathfrak{c}$ and nonzero $c_{i}, i=1, \ldots, k$. Observe that $f_{\xi} \neq 0$ on $\left[a_{n}, b_{n}\right], n \in B_{\xi}$. The sum $c_{1} f_{\xi_{1}}+\cdots+c_{k} f_{\xi_{k}}$ is nonzero on $\left[a_{n}, b_{n}\right]$ with $n \in B_{\xi_{1}} \cup \cdots \cup B_{\xi_{k}}$. From the Fichtenholz-Kantorovich theorem it follows that there exists $n_{0} \in B_{\xi} \backslash\left(B_{\xi_{1}} \cup \cdots \cup B_{\xi_{k}}\right)=B_{\xi}^{1} \cap B_{\xi_{1}}^{0} \cap \cdots \cap B_{\xi_{k}}^{0} \neq \emptyset$.

To finish the proof it is sufficient to show that all the linear combinations of functions $\left\{f_{\xi}: \xi<\mathfrak{c}\right\}$ also belong to $M$. Take $f=c_{1} f_{\xi_{1}}+c_{2} f_{\xi_{2}}$ for some $\xi_{1}, \xi_{2}<\mathfrak{c}$ and nonzero $c_{1}, c_{2} \in \mathbb{R}$. Then

$$
f(x)= \begin{cases}c_{1} F_{n}(x) & \text { for } x \in\left[a_{n}, b_{n}\right], n \in B_{\xi_{1}} \backslash B_{\xi_{2}} \\ c_{2} F_{n}(x) & \text { for } x \in\left[a_{n}, b_{n}\right], n \in B_{\xi_{2}} \backslash B_{\xi_{1}} \\ \left(c_{1}+c_{2}\right) F_{n}(x) & \text { for } x \in\left[a_{n}, b_{n}\right], n \in B_{\xi_{1}} \cap B_{\xi_{2}} \\ 0 & \text { otherwise. }\end{cases}
$$

Since $f$ is of the form (1), $f \in M$. In the same way we can show that any linear combination of functions $\left\{f_{\xi}: \xi<\mathfrak{c}\right\}$ belongs to $M$.

Hence to prove $\mathfrak{c}$-lineability of the classes of linearly sensitive functions described above it is sufficient to show that for these classes the assumptions of Theorem 2 are fulfilled.

A very similar method was used to prove $2^{\mathfrak{c}}$-lineability (or even algebrability) in the papers $[4,5,14]$.

\section{Applications}

In this section we will use the presented method to prove -lineability of families of functions linearly sensitive with respect to some known properties of real functions. We will start with the classic Luzin $(N)$-property introduced by Nikolai Luzin at the beginning of the twentieth century [10]. Recall that a function fulfills the Luzin $(N)$-property if the image of any null set has measure 
zero. The concept of the Luzin $(N)$-property plays an essential role in the theory of absolute continuity. A continuous function may not have Luzin $(N)$ property (for instance the Cantor function), but if a continuous function is of bounded variation and has the Luzin $(N)$-property then it is absolutely continuous (Banach-Zaretsky theorem).

In $1928 \mathrm{~S}$. Mazurkiewicz showed that there exists a real continuous function $f$ which fulfills the Luzin $(N)$-property such that $f+$ id does not have this property. Two years later Mazurkiewicz generalized this construction and proved the following theorem.

Theorem 4 (Mazurkiewicz [13]). There exists a continuous function $f$ : $[0,1] \rightarrow \mathbb{R}$ fulfilling the Luzin $(N)$-property such that $f+a$ id does not satisfy the Luzin $(N)$-property for any $a \neq 0$.

In fact, without any set-theoretic assumptions, Mazurkiewicz constructed a graph $Q$ of some function as an intersection of a sequence of closed subsets of $\mathbb{R}^{2}$. The projections of $Q$ on Ox and Oy are null sets while after transformation by adding $a \cdot$ id the projection of $Q$ on $\mathrm{Oy}$ is a set of positive measure. Making use of the Tietze theorem, the set $Q$ was complemented so that Mazurkiewicz obtained a graph of desired function defined on $[0,1]$.

We will use the above theorem to examine lineability of the family $\mathcal{A}_{N}$ of all continuous functions linearly sensitive to the Luzin $(N)$-property.

Theorem 5. The family $\mathcal{A}_{N}$ is c-lineable.

Proof. Let $F:[0,1] \rightarrow \mathbb{R}$ be a function from Mazurkiewicz's theorem. Take an arbitrary sequence of intervals $\left\{\left[a_{n}, b_{n}\right]: n \in \mathbb{N}\right\}$ such that

$$
0 \leq a_{1}<b_{1}<a_{2}<b_{2}<\cdots<1
$$

For any $n \in \mathbb{N}$ we divide the interval $\left[a_{n}, b_{n}\right]$ into three segments: $\left[a_{n}, c_{n}\right]$, $\left[c_{n}, d_{n}\right]$ and $\left[d_{n}, b_{n}\right]$. By squeezing and translating the function $F$ to the interval $\left[c_{n}, d_{n}\right]$, and adding two linear pieces, one can define a continuous function $F_{n}:\left[a_{n}, b_{n}\right] \rightarrow \mathbb{R}$ such that $F_{n}\left(a_{n}\right)=F_{n}\left(b_{n}\right)=0$. For example $F_{n}$ can be defined by the formula

$$
F_{n}(x)= \begin{cases}\frac{1}{n} \cdot F(0) \cdot \frac{x-a_{n}}{c_{n}-a_{n}} & \text { for } x \in\left[a_{n}, c_{n}\right] \\ \frac{1}{n} \cdot F\left(\frac{x-c_{n}}{d_{n}-c_{n}}\right) & \text { for } x \in\left[c_{n}, d_{n}\right] \\ \frac{1}{n} \cdot F(1) \cdot \frac{b_{n}-x}{b_{n}-d_{n}} & \text { for } x \in\left[d_{n}, b_{n}\right] .\end{cases}
$$

It is easy to check that $F_{n}$ has the Luzin $(N)$-property.

On the other hand, for any $a \neq 0$ the function $F_{a}(x):=F(x)+a x$ does not have $(N)$-property. Thus, there is a null set $X \subset[0,1]$ such that $F_{a}(X)$ is not a null set. Obviously, the set $Y:=\left(d_{n}-c_{n}\right) X+c_{n}$ has the measure zero and it is contained in $\left[c_{n}, d_{n}\right]$. Simultaneously, 


$$
\left(F_{n}+a \cdot i d\right)(Y)=\frac{1}{n} \cdot F_{a}(X)+a \cdot c_{n},
$$

so $\left(F_{n}+a \cdot i d\right)(Y)$ is not a null set, which proves that $F_{n}$ is linearly sensitive to $(N)$-property.

Now, fix a set $A \subset \mathbb{N}$ and a bounded sequence $\left(\alpha_{i}\right)_{i \in A}$ of nonzero numbers, and consider the function $G_{\alpha_{i}, A}$

$$
G_{\alpha_{i}, A}(x)= \begin{cases}\alpha_{i} F_{i}(x) & \text { if } x \in\left[a_{i}, b_{i}\right], i \in A \\ 0 & \text { if } x \in[0,1] \backslash \bigcup_{i \in A}\left[a_{i}, b_{i}\right] .\end{cases}
$$

Observe that $G_{\alpha_{i}, A}$ is continuous. It is evident when $A$ is a finite set. If $A$ is infinite, the continuity of $G_{\alpha_{i}, A}$ at $\sup A$ is the result of boundeness of $\left(\alpha_{i}\right)_{i \in A}$.

To complete the proof it is enough to show that $G_{\alpha_{i}, A}$ is linearly sensitive with respect to $(N)$-property. It is not difficult to see that $G_{\alpha_{i}, A}$ has $(N)$ property. Indeed, suppose that a set $X \subset[0,1]$ has measure zero. Since

$$
X=\bigcup_{i \in A}\left(X \cap\left[a_{i}, b_{i}\right]\right) \cup\left([0,1] \backslash \bigcup_{i \in A}\left[a_{i}, b_{i}\right]\right)
$$

and functions $F_{i}$ have $(N)$-property, the set

$$
G_{\alpha_{i}, A}(X)=\bigcup_{i \in A} \alpha_{i} F_{i}\left(X \cap\left[a_{i}, b_{i}\right]\right) \cup\{0\}
$$

has the measure zero, too.

Finally, fix a nonzero number $a$ and choose any $i \in A$. We know that the function $F_{i}(x)+a x$ does not have $(N)$-property, so there is a null set $Y \subset\left[a_{n}, b_{n}\right]$ such that the image of $Y$ under $F_{i}+a \cdot i d$ is not a null set. Since $\alpha_{i} \neq 0, G_{\alpha_{i}, A}(Y)$ is not a null set either.

Observe that we have proved the lineability on the maximum level, because there are only $\mathfrak{c}$ continuous real functions on $[0,1]$.

The Świątkowski condition was introduced in 1978 by Mańk and Świątkowski in the paper [11] connected with the sufficient conditions for the monotonicity of a function. They considered Darboux functions, Baire one functions and functions fulfilling some new condition, later called the Świątkowski condition (in [15]). They showed that those three conditions are independent and the sum of two functions with the Światkowski condition may not satisfy this condition. It is worth noting that any continuous function satisfies the Świątkowski condition.

Definition 6 ([11]). We say that $f$ satisfies the Światkowski condition if for all $x_{1}<x_{2}$ with $f\left(x_{1}\right) \neq f\left(x_{2}\right)$ there is a point $x \in\left(x_{1}, x_{2}\right)$ such that $f$ is continuous at $x$ and $f(x)$ lies between $f\left(x_{1}\right)$ and $f\left(x_{2}\right)$. 


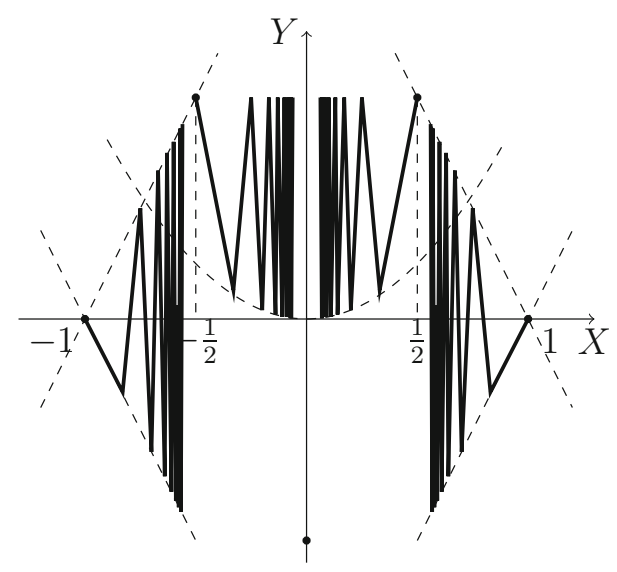

Figure 1. $y=F(x), F \in \mathcal{A}_{\dot{S}}$

Theorem 7. The family $\mathcal{A}_{S}$ of all Baire one functions linearly sensitive to the Światkowski condition is $\mathbf{c}$-lineable.

Proof. Let $\widetilde{F}:[0,1] \rightarrow \mathbb{R}$ be defined by the formula

$$
\widetilde{F}(x)= \begin{cases}-1 & \text { if } x=0 \\ 1 & \text { if } x=\frac{1}{2 k}, k=1,2,3, \ldots \\ x^{2} & \text { if } x=\frac{1}{2 k-1}, k=2,3, \ldots \\ 2-2 x & \text { if } x=\frac{1}{2}+\frac{1}{2 k}, k=1,2,3, \ldots \\ 2 x-2 & \text { if } x=\frac{1}{2}+\frac{1}{2 k-1}, k=1,2,3, \ldots \\ \text { affine } & \text { otherwise }\end{cases}
$$

and

$$
F(x)= \begin{cases}\widetilde{F}(x) & \text { for } x \in[0,1] \\ \widetilde{F}(-x) & \text { for } x \in[-1,0) .\end{cases}
$$

Evidently, $F$ (its graph is on Fig. 1) is a Baire one function as it has only three discontinuity points: at $-\frac{1}{2}$, at 0 and at $\frac{1}{2}$. Fix $x_{1}<x_{2}$. If $\left.F\right|_{\left[x_{1}, x_{2}\right]}$ is continuous then, by Darboux property, for any $y$ lying between points $F\left(x_{1}\right)$ and $F\left(x_{2}\right)$ there is a point $x \in\left(x_{1}, x_{2}\right)$ such that $F(x)=y$. Of course, $F$ is continuous at $x$. If $x_{1} \in\left[-1,-\frac{1}{2}\right)$ and $x_{2} \notin\left[-1,-\frac{1}{2}\right)$ then $F\left(\left(x_{1}, x_{2}\right)\right) \supset$ $F\left(\left(x_{1},-\frac{1}{2}\right)\right)=(-1,1)$, and again every value between $F\left(x_{1}\right)$ and $F\left(x_{2}\right)$ is achieved at some continuity point $x \in\left(x_{1}, x_{2}\right)$. If $x_{1} \in\left[-\frac{1}{2}, 0\right]$ and $x_{2} \in\left[0, \frac{1}{2}\right]$ then $F\left(\left(x_{1}, x_{2}\right)\right) \supset F\left(\left(x_{1}, 0\right)\right)=(0,1)$. Therefore, there is a point $x \in\left(x_{1}, x_{2}\right)$ such that $F$ is continuous at $x$ and $F(x)$ lies between $F\left(x_{1}\right)$ and $F\left(x_{2}\right)$ even if $x_{1}$ (or $x_{2}$ ) is equal to 0 . Finally, if $x_{2} \in\left(\frac{1}{2}, 1\right]$ and $x_{1} \notin\left(\frac{1}{2}, 1\right]$ then again $F\left(\left(x_{1}, x_{2}\right)\right) \supset(-1,1)$. Thus, $F$ satisfies the Świątkowski condition. 


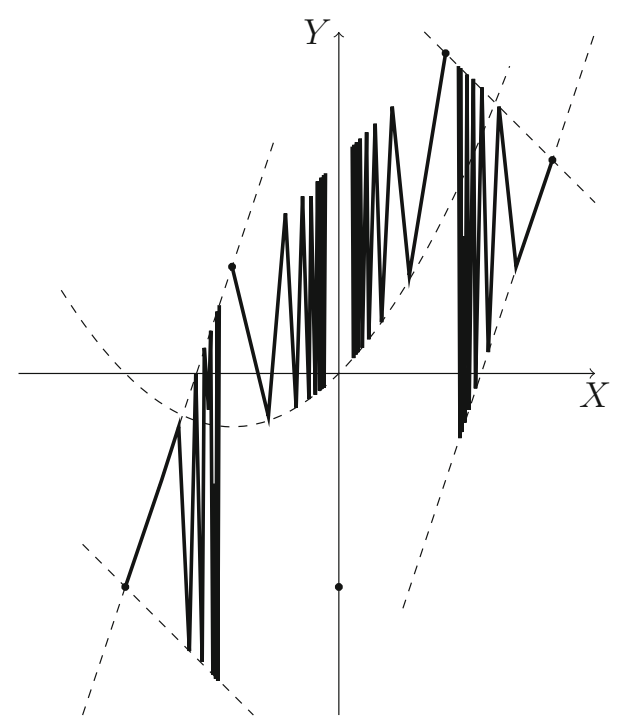

FiguRE 2. $y=F(x)+a x$ for $a>0$

Now, fix $a>0$. Note that the parabola $y=x^{2}+a x$ has the vertex at $-\frac{a}{2}$ (see Fig. 2). Therefore, for $k$ such that $\frac{1}{2 k-1}<\frac{a}{2}, x_{1}=-\frac{1}{2 k-1}$ and $x_{2}=0$ there is no $x \in\left(x_{1}, x_{2}\right)$ such that $F(x)<F\left(x_{1}\right)$. Hence $F+a$. id does not satisfy the Świątkowski condition. If $a<0$ the situation is symmetrical.

Analogously as in the proof of Theorem 5, we fix a sequence of intervals $\left\{\left[a_{n}, b_{n}\right]: n=1,2, \ldots\right\}$ such that

$$
0 \leq a_{1}<b_{1}<a_{2}<b_{2}<\cdots<1 .
$$

and observe that for any $n$ the function $F_{n}:\left[a_{n}, b_{n}\right] \rightarrow \mathbb{R}$ defined by the formula

$$
F_{n}(x)=F\left(\frac{2\left(x-a_{n}\right)}{b_{n}-a_{n}}-1\right)
$$

is a Baire one function linearly sensitive to the Światkowski condition and $F_{n}\left(a_{n}\right)=F_{n}\left(b_{n}\right)=0$.

It remains to prove that the function

$$
G_{\alpha_{i}, A}(x)= \begin{cases}\alpha_{i} F_{i}(x) & \text { if } x \in\left[a_{i}, b_{i}\right], i \in A \\ 0 & \text { if } x \in[0,1] \backslash \bigcup_{i \in A}\left[a_{i}, b_{i}\right]\end{cases}
$$

is a Baire one function linearly sensitive to Świątkowski condition, for any set $A \subset \mathbb{N}$ and any bounded sequence $\left(\alpha_{i}\right)_{i \in A}$ of nonzero numbers. Since $G_{\alpha_{i}, A}$ has at most countably many discontinuity points, it is a Baire one function. It is also easy to check that for any $a \neq 0$ the function $G_{\alpha_{i}, A}+a \cdot$ id does 
not fulfill the Światkowski condition. The only remaining point concerns the Swiatkowski condition of the function $G_{\alpha_{i}, A}$.

Assume that $0 \leq x_{1}<x_{2} \leq 1$ and $\left|G_{\alpha_{i}, A}\left(x_{1}\right)\right|<\left|G_{\alpha_{i}, A}\left(x_{2}\right)\right|$. Since $\left|G_{\alpha_{i}, A}\left(x_{2}\right)\right|>0$, there exists $n \in N$ such that $x_{2} \in\left(a_{n}, b_{n}\right)$. If $x_{1}$ and $x_{2}$ belong to the same interval then the thesis follows from the fact that $F_{n}$ fulfills the Światkowski condition. Suppose that $x_{1} \notin\left(a_{n}, b_{n}\right)$. If $G_{\alpha_{i}, A}$ is continuous on $\left[a_{n}, x_{2}\right]$ then $G_{\alpha_{i}, A}$ takes all values between 0 and $G_{\alpha_{i}, A}\left(x_{2}\right)$. In particular, it takes values between $G_{\alpha_{i}, A}\left(x_{1}\right)$ and $G_{\alpha_{i}, A}\left(x_{2}\right)$.

If there is a discontinuity point of $G_{\alpha_{i}, A}$ between $a_{n}$ and $x_{2}$, then $G_{\alpha_{i}, A}\left(\left(a_{n}, x_{2}\right)\right)=\alpha_{n} F_{n}\left(\left(a_{n}, x_{2}\right)\right)=\left(-\left|\alpha_{n}\right|,\left|\alpha_{n}\right|\right)$. Moreover, $\left.G_{\alpha_{i}, A}\right|_{\left(a_{n}, a_{n}+\frac{b_{n}-a_{n}}{4}\right)}$ is continuous,

$$
G_{\alpha_{i}, A}\left(\left(a_{n}, a_{n}+\frac{b_{n}-a_{n}}{4}\right)\right)=\left(-\left|\alpha_{n}\right|,\left|\alpha_{n}\right|\right)
$$

and $0 \leq\left|G_{\alpha_{i}, A}\left(x_{1}\right)\right|<\left|G_{\alpha_{i}, A}\left(x_{2}\right)\right| \leq\left|\alpha_{n}\right|$. If $\left|G_{\alpha_{i}, A}\left(x_{2}\right)\right|<\left|G_{\alpha_{i}, A}\left(x_{1}\right)\right|$ the considerations are analogous.

In the paper [17] Wódka proved that the family of all functions satisfying the Świątkowski condition is not $\mathfrak{c}^{+}$-lineable, which means that even in the wider class (not only Baire one) of functions satisfying the Światkowski condition the obtained result is on the best possible level.

Let us look closer to the sensitivity of Darboux functions. Kirchheim and Natkaniec [9] proved that if the union of fewer then $\mathfrak{c}$ of the first category subsets of $\mathbb{R}$ is a set of the first category $(\operatorname{add}(\mathcal{M})=\mathfrak{c})$, then there exists a Darboux function $F$ such that $F+f$ is not Darboux for any continuous and nowhere constant function $f$. Based on the general method one can prove that the family of linearly sensitive functions with respect to the Darboux property is $\mathfrak{c}$-lineable. On the other hand, if $F$ is a Baire one Darboux function and $f$ is continuous, then $F+f$ is a Baire one Darboux function (see [7]). Consequently, there is no Baire one function sensitive to the Darboux property.

Now we will consider a property which is a bit stronger than the Darboux property. It was introduced by Maliszewski [12] in 1995 as a modification of the Świątkowski condition.

Definition 8. We say that $f$ satisfies the strong the Świątkowski condition if for all $x_{1}<x_{2}$ with $f\left(x_{1}\right) \neq f\left(x_{2}\right)$ and each $y$ between $f\left(x_{1}\right)$ and $f\left(x_{2}\right)$, there is a point $x \in\left(x_{1}, x_{2}\right)$ such that $f(x)=y$ and $f$ is continuous at $x$.

Theorem 9. The family $\mathcal{A}_{s S}$ of all Baire one functions linearly sensitive to strong Światkowski condition is $\mathfrak{c}$-lineable. 


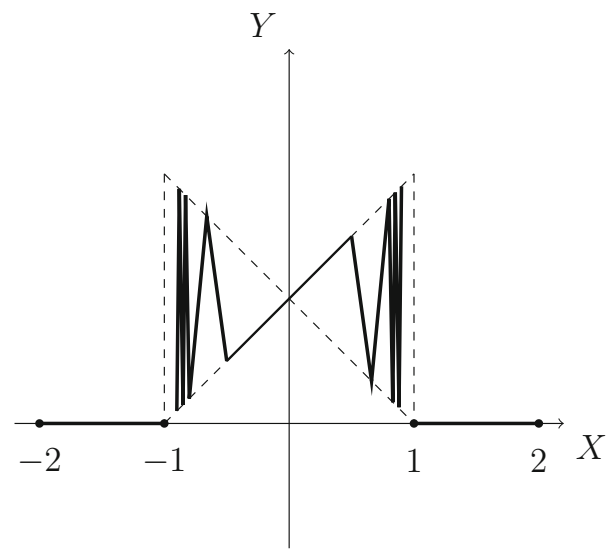

Figure 3. $y=F(x)$

Proof. Firstly consider the function $F:[-2,2] \rightarrow \mathbb{R}$

$F(x)= \begin{cases}0 & \text { for } x \in[-2,-1] \cup[1,2] \\ x+1 & \text { for }|x|=1-\frac{1}{2 k} \text { or } x \in\left[-\frac{1}{2}, \frac{1}{2}\right] \\ -x+1 & \text { for }|x|=1-\frac{1}{2 k-1} \\ \text { affine } & \text { on intervals }\left[1-\frac{1}{k}, 1-\frac{1}{k+1}\right],\left[\frac{1}{k-1}-1, \frac{1}{k}-1\right] \text { for } k>2 .\end{cases}$

It is clear that $F$ is a Darboux Baire one function. It satisfies the strong Świątkowski condition because $F$ is discontinuous only at -1 and 1 , and

$$
F(1)=F(-1)=0=\min \{F(x): x \in[-2,2]\} .
$$

Indeed, take any $x_{1}<x_{2}$ and $y$ between $F\left(x_{1}\right)$ and $F\left(x_{2}\right)$. Since $F$ is Darboux, there is $x \in\left(x_{1}, x_{2}\right)$ such that $F(x)=y$. Since $F(x)>0, F$ is continuous at $x$ (see Fig. 3).

However, for any $a>0$ the function $F+a \cdot$ id does not satisfy the strong Świątkowski condition. Indeed, the value $-a$ is taken only at discontinuity point of $F+a \cdot$ id (Fig. 4). For $a<0$ the situation is analogous. Thus, $F$ is linearly sensitive with respect to the strong Świ ątkowski condition.

Now, fix a sequence $\left\{\left[a_{n}, b_{n}\right]: n=1,2, \ldots\right\}$ with $0 \leq a_{1}<b_{1}<a_{2}<$ $b_{2}<\cdots<1$. For any $n \in \mathbb{N}$ the function $F_{n}:\left[a_{n}, b_{n}\right] \rightarrow \mathbb{R}$ defined by the formula

$$
F_{n}(x)=F\left(\frac{4\left(x-a_{n}\right)}{b_{n}-a_{n}}-2\right)
$$

is Baire one and $F_{n}\left(a_{n}\right)=F_{n}\left(b_{n}\right)=0$. It is not difficult to check that $F_{n}$ is linearly sensitive to the strong Świątkowski condition. 


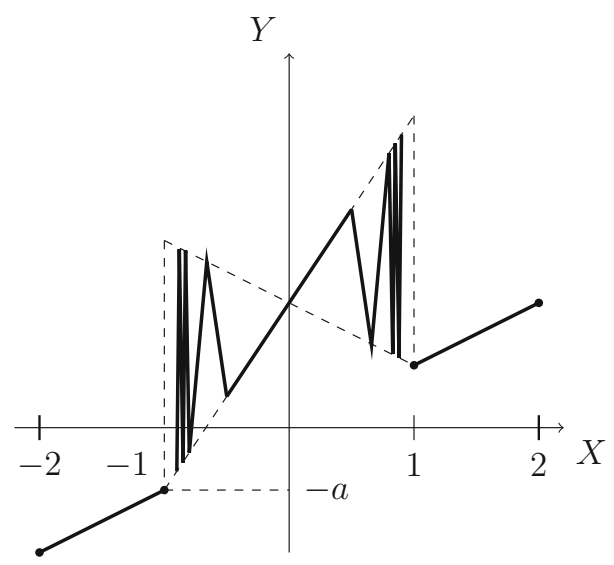

Figure 4. $y=F(x)+a x$ for $a>0$

For a fixed set $A \subset \mathbb{N}$ and a bounded sequence $\left(\alpha_{i}\right)_{i \in A}$ of nonzero numbers, consider the function $G_{\alpha_{i}, A}$

$$
G_{\alpha_{i}, A}(x)= \begin{cases}\alpha_{i} F_{i}(x) & \text { if } x \in\left[a_{i}, b_{i}\right], i \in A \\ 0 & \text { if } x \in[0,1] \backslash \bigcup_{i \in A}\left[a_{i}, b_{i}\right]\end{cases}
$$

It is clear that $G_{\alpha_{i}, A}$ is a Baire one function. To observe that $G_{\alpha_{i}, A}$ fulfills the strong Świątkowski condition fix $x_{1}, x_{2} \in[0,1]$. If both points belong to the same interval $\left[a_{i}, b_{i}\right]$ (or at least one of them belongs to $[0,1] \backslash \bigcup_{i \in A}\left[a_{i}, b_{i}\right]$ ) then the existence of an appropriate point $x$ follows from the fact that $\alpha_{i} F_{i}$ satisfies the strong Świątkowski condition. So let us assume that $x_{1} \in\left[a_{i_{1}}, b_{i_{1}}\right]$ and $x_{2} \in\left[a_{i_{2}}, b_{i_{2}}\right]$ for $i_{1} \neq i_{2}$. Then any value between $G_{\alpha_{i}, A}\left(x_{1}\right)$ and 0 is achieved at continuity point of $\alpha_{i_{1}} F_{i_{1}}$, and any value between $G_{\alpha_{i}, A}\left(x_{2}\right)$ and 0 is achieved at continuity point of $\alpha_{i_{2}} F_{i_{2}}$.

Finally, consider the function $G_{\alpha_{i}, A}+a \cdot$ id for some nonzero $a$. The fact that this function does not satisfy the strong Światkowski condition follows from the analogous property of functions $F_{i}+a \cdot$ id for $i \in A$.

Again, it is the lineability on the maximum level. Moreover, according to the paper [17], the last result is the best possible in the class of all functions linearly sensitive with resspect to the strong Świątkowski condition.

Observe, that in the case of Luzin $(N)$-property we did not need the formula for a function. We could use any function which is sensitive with respect to $(N)$-property. When we consider the Światkowski condition, the form of the function plays a crucial role. It is not enough to use any function linearly sensitive with respect to Świątkowski condition. 
Indeed, the function $H$ defined by the formula

$$
H(x)= \begin{cases}0 & \text { for } x \in\left[-1,-\frac{1}{2}\right] \cup\left[\frac{1}{2}, 1\right] \text { and }|x|=\frac{1}{2 k}, k \in \mathbb{N} \\ -2 & \text { for } x=0 \\ x^{2}-1 & \text { if }|x|=\frac{1}{2 k-1}, k \in \mathbb{N} \\ \text { affine } & \text { on the intervals }\end{cases}
$$

is linearly sensitive with respect to the Świątkowski condition (see Figs. 5, 6).

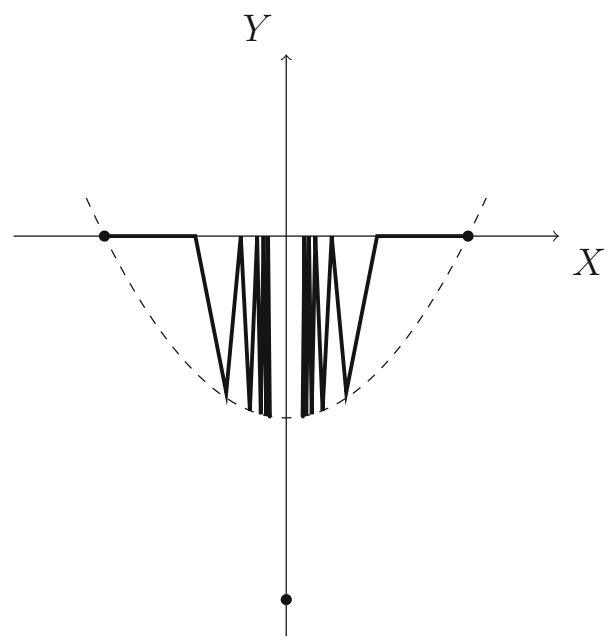

FiguRE 5. $y=H(x)$

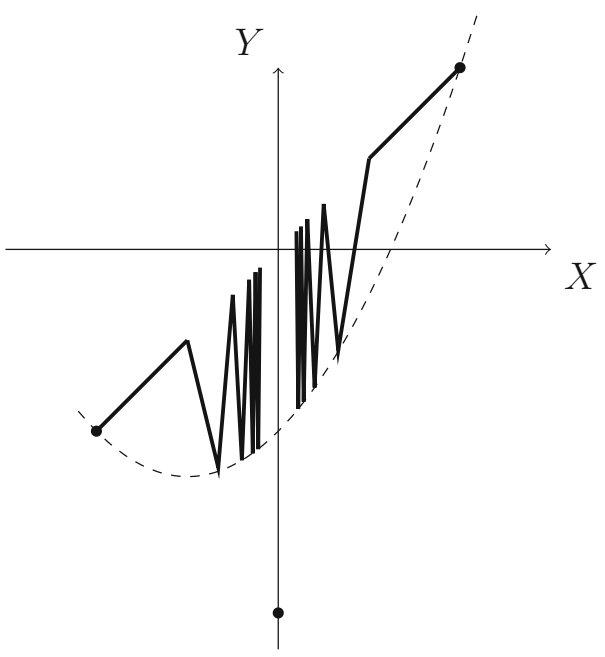

Figure 6. $y=H(x)+a x$, for $a>0$ 


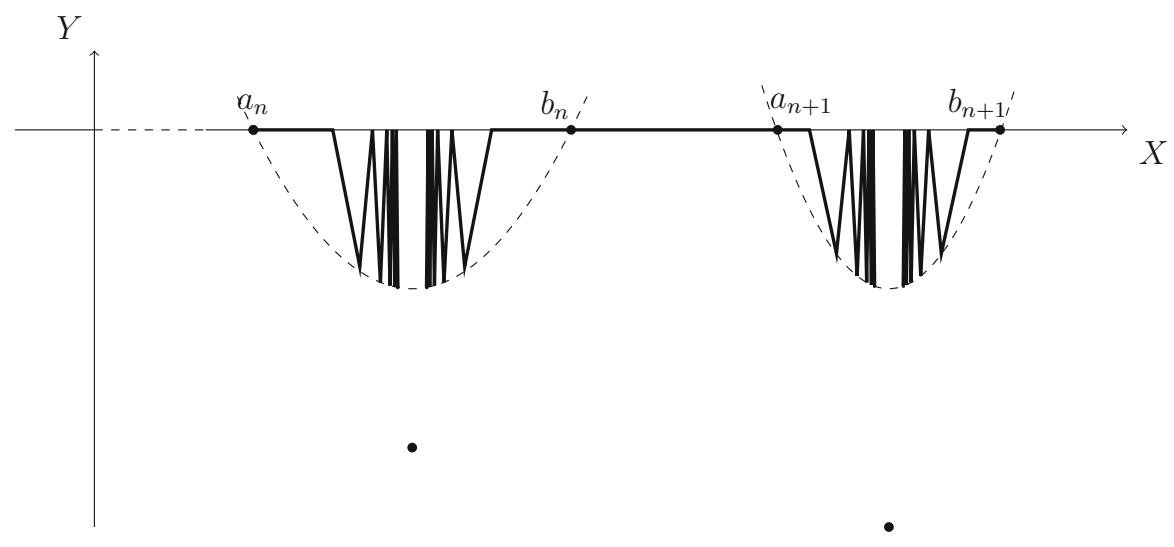

Figure 7. Functions of type $H$ on two disjoint intervals $\left[a_{n}, b_{n}\right]$ and $\left[a_{n+1}, b_{n+1}\right]$

However, for two disjoint intervals the sum of functions obtained from $H$ by squeezing and translating (and connected by $y=0$ on $\left[b_{n}, a_{n+1}\right]$ ) may not satisfy the Świątkowski condition as we can observe on Fig. 7.

It is enough to take $x_{1}=\frac{a_{n}+b_{n}}{2}$ and $x_{2}=\frac{a_{n+1}+b_{n+1}}{2}$. Then the obtained function has the values: -1 at $x_{1}$ and -2 at $x_{2}$, and the Światkowski condition is not satisfied. So the function must be chosen in such a way that for any disjoint intervals the sum of such functions must still fulfill the Świątkowski condition.

Open Access. This article is licensed under a Creative Commons Attribution 4.0 International License, which permits use, sharing, adaptation, distribution and reproduction in any medium or format, as long as you give appropriate credit to the original author(s) and the source, provide a link to the Creative Commons licence, and indicate if changes were made. The images or other third party material in this article are included in the article's Creative Commons licence, unless indicated otherwise in a credit line to the material. If material is not included in the article's Creative Commons licence and your intended use is not permitted by statutory regulation or exceeds the permitted use, you will need to obtain permission directly from the copyright holder. To view a copy of this licence, visit http://creativecommons.org/ licenses/by $/ 4.0 /$.

\section{References}

[1] Aron, R., Guriaryi, V.I., Seoane, J.B.: Lineability and spaceability of sets of functions on $\mathbb{R}$. Proc. Am. Soc. 133(3), 795-803 (2005)

[2] Aron, R., Garcia, D., Maestre, M.: Linearity in non-linear problems. RACSAM. Rev. R. Acad. Cienc. Exactas Fs. Nat. Ser. A Mat. 95(1), 7-12 (2002) 
[3] Aron, R.M., González, L.B., Pellegrino, D.M., Sepúlveda, J.B.S.: Lineability: The Search of Lineability in Mathematics. Monographs and Research Notes in Mathematics. CRC Press, Boca Raton (2016)

[4] Bartoszewicz, A., Bienias, M., Głąb, S.: Independent Bernstein sets and algebraic constructions. J. Math. Anal. Appl. 393, 138-143 (2012)

[5] Bartoszewicz, A., Głąb, S., Pellegrino, D., Seoane-Sepúlveda, J.B.: Algebrability, non-linear properties and special functions. Proc. Am. Math. Soc. 141(10), 33913402 (2013)

[6] Bernal-González, L., Pellegrino, D.M., Seoane-Sepúlveda, J.B.: Linear subsets of nonlinear sets in topological vector spaces. Bull. Am. Math. Soc. 51, 71-130 (2014)

[7] Bruckner, A.B.: Differentiation of Real Functions. Springer, Berlin (1978)

[8] Fichtenholz, G.M., Kantorovich, L.V.: Sur le opérations linéares dans l'espace de fonctions bornées. Studia Math. 5, 69-98 (1934)

[9] Kirchheim, B., Natkaniec, T.: On universally bad Darboux functions. Real Anal. Exch. 16, 481-486 (1990-1991)

[10] Luzin, N.N.: The Integral and Trigonometric Series. GITTL, Moscow-Leningrad (1915). (in Russian)

[11] Mańk, T., Świątkowski, T.: On some class of functions with Darboux's characteristic. Zeszyty Nauk. Politech. Łódz. Mat. 11, 5-10 (1978)

[12] Maliszewski, A.: On the limits of strong Świątkowski functions. Zeszyty Nauk. Politech. Łódz. Mat. 27(719), 87-93 (1995)

[13] Mazurkiewicz, S.: Sur les fonctions qui satisfont à la condition $(N)$. Fund. Math. 16(1), 348-352 (1930)

[14] Natkaniec, T.: Algebrability of some families of Darboux-like functions. Linear Algebra Appl. 439(10), 3256-3263 (2013)

[15] Pawlak, H., Wilczyñski, W.: On the condition of Darboux and Świątkowski for functions of two variables. Zeszyty Nauk. Politech. Eódz. Mat. No. 15, 31-35 (1982)

[16] Seoane, J.B.: Chaos and lineability of pathological phenomena in analysis. Thesis (Ph.D), Kent State University. ISBN: 987-0542-78798-0 (2006)

[17] Wódka, J.: Subsets of some families of real functions and their algebrability. Linear Algebra Appl. 459, 454-464 (2014) 
Artur Bartoszewicz and Małgorzata Terepeta

Institute of Mathematics

Lodz University of Technology

ul. Wólczańska 215

90-924 Lodz

Poland

e-mail: artur.bartoszewicz@p.lodz.pl

Małgorzata Terepeta

e-mail: malgorzata.terepeta@p.lodz.pl

Małgorzata Filipczak

Faculty of Mathematics and Computer Science

Łódź University

ul. Stefana Banacha 22

90-238 Lodz

Poland

e-mail: malgorzata.filipczak@wmii.uni.lodz.pl

Małgorzata Terepeta

Center of Mathematics and Physics, Lodz University of Technology al. Politechniki 11

90-924 Lodz

Poland

Received: October 25, 2019.

Accepted: March 14, 2020.

Publisher's Note Springer Nature remains neutral with regard to jurisdictional claims in published maps and institutional affiliations. 